ICRES 2019: International Conference on Robot

Ethics and Standards, London, UK, 29-30 July 2019.

https://doi.org/10.13180/icres.2019.29-30.07.016

\title{
ETHICAL IMPLICATIONS OF EMBODIED PROSTHETIC DEVICES
}

\author{
B. WARD-CHERRIER \\ Department of Engineering Mathematics, University of Bristol \\ Bristol, United Kingdom \\ *E-mail: b.ward-cherrier@bristol.ac.uk \\ A. VAN MARIS \\ Bristol Robotics Laboratory, University of the West of England \\ Bristol, United Kingdom \\ E-mail: anouk.vanmaris@uwe.ac.uk
}

\section{Introduction}

As research in prosthetics explores the use of improved sensory feedback techniques ${ }^{1}$ and control methods, ${ }^{2}$ the capabilities of prostheses are becoming more comparable to biological limbs. This development process, guided by prosthetic device users, ${ }^{3}$ is gradually leading to a feeling of embodiment in which the user sees the prosthetic device as being a part of their own body. ${ }^{4}$

The embodiment of prosthetic devices clearly has strong advantages for amputees, allowing for better integration of the device, a more natural use of prostheses, ${ }^{5}$ and minimizing the sense of difference associated with the use of a prosthetic device. ${ }^{6}$ However, a fully embodied prosthesis raises potential ethical concerns linked to the design and development of such devices, which we explore in the following section.

\section{Proposed Research Questions}

Although there are a number of valid ethical concerns which apply to prosthetic devices generally (for instance privacy, governance, and human enhancement technologies), we focus here on topics specifically related to the embodiment of prostheses and identify two research questions for further investigation:

'Does embodiment lead to overtrusting the prosthetic device?' Both embodied and non-embodied prosthetic devices could be beneficial to amputees, ${ }^{7}$ with embodied devices providing a more natural and seamless experience in activities of daily living. ${ }^{5}$ However, these fully embodied devices do not necessarily have the same material properties or functional capabilities as a biological limb. If the user feels that the device is corporeal, they may rely on it in situations where it does not meet their needs. For instance, in an emergency situation, the user may use the artificial limb for quick, accurate grasping which may be beyond the current functional capabilities of the device and could result in harm to the user. This phenomenon could be close to occurring in current neural prostheses, with users finding these can provide 'the functional and psychosocial experience of having a hand again'. ${ }^{5}$ Therefore, it is important to find a balance between improving the functionality of the device and increasing the feeling of embodiment, such that the trust in the device is at a level which reflects its functional capabilities.

'Should a feeling of pain be included in embodied prosthetic devices?' Ad- 
ditionally, there is a question of how faithful to biology prosthetic designs should become to achieve a feeling of embodiment. The sense of nociceptive pain clearly has evolutionary advantages in biological systems, functioning as a warning signal for potential harm. Artificial prosthetic devices could employ a similar pain mechanism to signal possible damage to the device or an imminent danger to the user. A recent study has demonstrated how such feedback could be implemented in an electronic skin integrated within a prosthetic hand. ${ }^{8}$ However, these kinds of devices lead to ethical issues around the concept of inflicting painful stimuli to users, which could even be seen as a violation of the Hippocratic oath. The risks linked to potential malfunctioning are also particularly high with these devices, since painful signals could accidentally be delivered to the user in non-harmful situations, creating an artificial feeling of chronic pain. Conversely, a fully embodied device with no pain signalling raises issues to do with possible over-reliance or overtrust in the device. The lack of pain feedback in these devices increases the chances of the user unknowingly being in a dangerous situation. The asymmetry between artificial and biological limbs could also produce confusion in emergency situations, leading to possible harm to the user as well as damage to the prosthetic device. A lack of pain would also make the device more likely to be used for malicious reasons, such as harming others. It could in effect become a kind of embodied tool, with the high levels of feedback and control associated with human limbs, yet without the limiting pain signals.

Current and future research in prosthetics is likely to raise ethical concerns regarding the embodiment of devices. We have proposed two research questions to help guide the development and use of embodied prostheses: 'Does embodiment lead to overtrusting the prosthetic device?' and 'Should a feeling of pain be included in embodied prosthetic devices?'. These questions are essential to the development of ethically safe and trustworthy embodied prosthetic devices.

\section{References}

1. C. Antfolk, M. Dalonzo, B. Rosén, G. Lundborg, F. Sebelius and C. Cipriani, Expert review of medical devices 10, 45 (2013).

2. C. Cipriani, F. Zaccone, S. Micera and M. C. Carrozza, IEEE Transactions on Robotics 24, 170 (2008).

3. S. Lewis, M. F. Russold, H. Dietl and E. Kaniusas, User demands for sensory feedback in upper extremity prostheses, in 2012 IEEE International Symposium on Medical Measurements and Applications Proceedings, 2012.

4. P. D. Marasco, K. Kim, J. E. Colgate, M. A. Peshkin and T. A. Kuiken, Brain 134, 747 (2011).

5. E. L. Graczyk, L. Resnik, M. A. Schiefer, M. S. Schmitt and D. J. Tyler, Scientific reports 8, p. 9866 (2018).

6. A. Saradjian, A. R. Thompson and D. Datta, Disability and Rehabilitation 30, 871 (2008).

7. C. D. Murray, Disability and Rehabilitation 26, 963 (2004).

8. L. E. Osborn, A. Dragomir, J. L. Betthauser, C. L. Hunt, H. H. Nguyen, R. R. Kaliki and N. V. Thakor, Science Robotics 3, p. eaat3818 (2018). 